ISSN electrónico: 2172-9077

DOI: https://doi.org/10.14201/fjc-v22-22696

\title{
ANÁLISIS DEL IMPACTO DE LAS REDES SOCIALES SOBRE EL TRÁFICO WEB DE LOS CIBERMEDIOS NATIVOS DIGITALES ESPAÑOLES
}

\section{Analysis of the impact of social networks on Spanish digital native cyber-media web traffic}

\section{Dr. David PARRA VALCARCE}

Profesor titular, Universidad Complutense de Madrid, España

E-mail: davidparra@ccinf.ucm.es

(iD) https://orcid.org/0000-0003-1811-7525

\author{
Lda. Charo ONIEVA MALLERO \\ Universidad Complutense de Madrid, España \\ E-mail: onieva@vivavoz.net \\ (iD) https://orcid.org/0000-0002-0485-0876
}

Fecha de recepción del artículo: 15/03/2020

Fecha de aceptación definitiva: 30/03/2020

\begin{abstract}
RESUMEN
La apuesta de los cibermedios nativos digitales por las redes sociales como instrumentos estratégicos para incrementar el volumen de tráfico web hacia sus portales se sustenta en aspectos como la consolidación de la web semántica manual o la eclosión de los prosumidores. Este artículo se plantea como objetivo principal analizar hasta qué punto es cierto que dichas plataformas contribuyen a incrementar el número de visitantes y si el esfuerzo que realiza la industria de la información para formar parte de ellas está o no justificado. Se examina dicha cuestión a partir de la selección de una muestra significativa de diez de ellos basada en el criterio cuantitativo del número mensual de usuarios únicos que acceden a sus sitios web, empleando para el estudio un software como SimilarWeb. Asimismo, se lleva a cabo una comparación entre el impacto de las redes sociales y el que tienen magnitudes como el tráfico directo o el uso de buscadores basados en procedimientos de posicionamiento SEO como elementos de generación de tráfico web total.

Palabras clave: Cibermedios; redes sociales; prosumidor; comunicación digital; periodismo.
\end{abstract}

\begin{abstract}
The commitment of digital native cybermedia for social networks as strategic instruments to increase the volume of web traffic to their portals is based on aspects such as the consolidation of the manual semantic web or the emergence of prosumers. The main purpose of this article is to analyze to what extent it is true that these platforms contribute to in-creasing the number of visitors and whether or not the effort made by the information
\end{abstract}


industry in order to be part of them is justified. This question is examined by means of the selection of a significant sample of ten of them based on the quantitative criteria of unique user monthly numbers who access their websites, using a piece of software like Simi-larWeb for the study. Likewise, a comparison is made between the impact of social networks and the impact of magni-tudes such as direct traffic or the use of search engines based on SEO positioning procedures as elements of total web traffic generation.

Keywords: Cybermedia; social networks; prosumer; digital communication; journalism.

\section{Introducción}

Identificamos los cibermedios nativos digitales con aquellos que «operan exclusivamente en la red y no dependen, ni editorial ni financieramente, de ninguna matriz impresa o audiovisual" (García Avilés y González Esteban, 2013, p. 154). Desde tal punto de vista, a lo largo de los últimos veinte años son ya tres las generaciones de cibermedios nativos digitales que coexisten en el mercado, tanto a escala internacional como en el caso concreto de España.

La primera de ellas aparece en la segunda mitad de la última década del siglo XX, un momento caracterizado por aspectos como la gradual eclosión de internet, convertida en un formidable almacén de toda clase de contenidos, el escaso entusiasmo con que una parte apreciable de los empresarios de la información contempla las posibilidades de la red para la distribución y explotación comercial de sus productos informativos y los deficientes niveles de calidad de acceso padecidos por los usuarios debido a la superposición de diferentes tecnologías de comunicaciones analógicas y digitales.

Forman parte de esta primera generación de pioneros en la red cibermedios nativos digitales como Vilaweb, creado en 1996 y considerado como el primero de todos ellos, o Estrella Digital, aparecido en 1998 y que aporta el rasgo diferencial de optar por una temática de ámbito general frente a la apuesta regional del primero de ellos. Ambos continúan en la actualidad, aquél con una sólida propuesta de contenidos y una acertada explotación comercial de nicho que ha sido examinada por autores como Iglesias y González Díaz (2012) y Hernández Soriano y Cristóbal (2014), y éste sujeto a diversas vicisitudes en forma de cambios de accionariado y bandazos ideológicos.

El comienzo del siglo XXI sienta las bases para el surgimiento en España de la segunda generación de nativos digitales. La convicción de la irreversibilidad del cambio tecnológico que supone una realidad como internet para la práctica totalidad de los sectores productivos (y también para la industria de la información), el continuado crecimiento de la base de internautas dispuestos al consumo de toda clase de contenidos online, la drástica caída de las tarifas de conexión y la gradual mejora en la calidad de los accesos constituyen algunos de los rasgos característicos de este momento.

Se encuadran dentro de esa segunda generación proyectos sumamente variados en facetas como línea editorial, temática, estructura societaria o tecnologías de navegación. No obstante, comparten, con alguna excepción muy notable, una formulación de modelo de negocio sustentada en la gratuidad de la totalidad (o, al menos, la inmensa mayoría) de sus contenidos y su financiación prioritaria a través de la publicidad.

Entre las cabeceras más relevantes por el número de usuarios únicos logrado y/o el grado de notoriedad alcanzado se encuentran Libertad Digital (creado en 2000), 
El Confidencial (2001), Periodista Digital (2004), El Plural (2005) o La Información (2009), todos ellos activos hoy en día, junto a proyectos extintos como Soitu (2007) o Factual (2009), una novedosa iniciativa que a diferencia de las anteriores abogaba por contenidos exclusivamente dirigidos a sus suscriptores.

La literatura científica ha analizado algunos de estos proyectos, destacando los trabajos de González Esteban (2010) sobre Soitu, Salaverría (2010) que de manera tangencial examina los primeros pasos de La Información, así como Mayoral y Edo (2014), Vara (2016) o Vázquez Herrero (2018) referidos a El Confidencial.

A partir del año 2011 nos adentramos en la tercera generación de cibermedios nativos digitales. Advertimos cuatro características distintivas respecto a los de anteriores etapas: la consolidación de la tendencia a acceder a todo tipo de contenidos, lo que para la industria de la información implica una significativa tarea de adecuación al soporte formal en forma del conocido como diseño responsivo o adaptativo (Gardner, 2011, p. 14); la transformación de internet en una mega ágora que facilita la generación y compartición de documentación e información; el empleo sistemático dentro de su estructura productiva de algoritmos, entendidos como un conjunto de códigos de software (Monasterio, 2017, p. 186), construcciones matemáticas (Hill, 2016, p. 39) y elementos robóticos (Steiner, 2012, p. 22); y la amplia variedad de modelos de negocio adoptados.

Se asiste a una considerable proliferación de proyectos de una amplia gama, ateniéndose tanto a los nueve criterios de distinción (plataforma, temporalidad, tema, alcance, titularidad, autoría, enfoque, finalidad económica y dinamismo) esbozados por Salaverría (2017, p. 24) como a las notables diferencias en aspectos como modelo de negocio, relación con las audiencias o apuesta estratégica por determinados géneros periodísticos establecidas por Parra (2017, p. 431).

Algunos de los casos más significativos que advertimos dentro de la tercera generación de cibermedios nativos digitales son Voz Pópuli (fundado en 2011), El Diario (2012), Huffington Post (2012) como franquicia del original estadounidense, Infolibre (2013), Contexto y Acción (CTXT) (2015), El Español (2015), El Independiente (2015), Es Diario (2015) y OK Diario (2015), todos actualmente operativos, a los que habría que añadir el ya desaparecido Bez Diario (2015).

La entrada en el tercer milenio conlleva, entre otras circunstancias, una notable mutación en la concepción de internet, tal y como se anticipaba con anterioridad. Si hasta ese entonces se la identificaba con un gigantesco almacén de todo tipo de contenidos a partir de ese instante se la equipara con la denominada web semántica manual o web 2.0 en la terminología explicitada por Di Nucci (1999, p. 32), quien considera que la red de redes supone una vía estratégica para mostrar cómo la web a la que estaban habituados los usuarios se transforma tanto en sus interfaces como en la visualización de sus contenidos ocasionando un profundo cambio en la experiencia de los internautas y en sus procedimientos de interactuación.

Esta idea inicial fue posteriormente conceptualizada por O'Reilly (2005) al definir esta novedosa internet como una mega ágora donde los internautas de todo el mundo establecen una charla a escala global en la que comparten conocimientos, inquietudes y experiencias, al tiempo que ese proceso colaborativo conforma un nuevo escenario de relación entre las corporaciones de toda índole y sus audiencias que marca un antes y un después en todo lo que atañe a la transmisión de contenidos.

Los trabajos de ambos investigadores fueron completados con numerosas aportaciones desde variadas perspectivas: esencialmente tecnológica, tanto en su faceta 
de investigación básica como aplicada (Kamel Boulos y Wheeler, 2007; Coleman y Levine, 2008; Bofil, 2013; o Caruso, 2018); y en el ámbito de la educación y la formación (Andersen, 2007; Grosseck, 2009; McHaney, 2012; o Harris y Rea, 2019).

A ellas debemos añadir las centradas específicamente en el campo de la comunicación. Para Briggs (2007, p. 33) esta novedosa noción de internet se identifica con sitios web convertidos en plataformas de computación que ofrecen sus aplicaciones a los usuarios finales al tiempo que Fumero y Roca (2007, p. 10) consideran que deviene en un espacio con cabida para los agentes sociales. Por su parte Cebrián (2010, p. 18) enfatiza el hecho de que se asiste a un entorno emergente que apuesta por la interactividad de las audiencias mientras que Mcnamara (2013) centra su análisis en el rol que desempeñan como herramientas de dinamización de acciones de comunicación.

Blogs, canales audiovisuales, portales de fotografía y música, plataformas educativas, redes sociales, servicios de sindicación de contenidos, wikis y podcasts constituyen algunos de los elementos de esa emergente web 2.0 donde surge la figura del prosumer o prosumidor como síntesis de productor y consumidor y en oposición a la tradicional consideración del receptor pasivo característico de la tradicional industria de los medios de comunicación de masas.

Este prosumidor es descrito como el sujeto paradigmático de la tercera ola, entendida como un entorno que supera las habituales barreras geográficas características de las sociedades industrialmente avanzadas y auspicia el fenómeno de la globalización (Toffler, 1980) e identificado con aquella persona que hace las cosas a su manera sin dejarse influir por las grandes corporaciones que deben adaptarse a este nuevo contexto (Tapscott, 1995).

De este modo quedan establecidos un conjunto de procedimientos particularmente adaptados a las pautas de consumo globales y de comunicación e información de dichos prosumidores no sólo a la hora de llevar a cabo la distribución de los contenidos sino incluso en lo que se refiere a la propia generación de los mismos, en el que una innovación como las redes sociales adquiere particular relevancia (Bird, 2011).

Por consiguiente ese cambio conceptual de internet en combinación con los avances en el ámbito de las tecnologías de la información y las comunicaciones en facetas como el desarrollo de nuevas plataformas hardware y programas y aplicaciones software de la más variada índole o el diseño y lanzamiento al mercado de nuevos dispositivos portátiles entre los que tabletas electrónicas y teléfonos móviles inteligentes alcanzan particulares niveles de implantación sientan las bases para que la industria de los medios de comunicación en general y los cibermedios nativos digitales en particular consideren las redes sociales como una herramienta estratégica de particular utilidad a la hora de generar tráfico adicional a sus correspondientes portales web.

\section{Objetivos e hipótesis}

La base fáctica de estas novedosas plataformas se sitúa a finales de los años veinte del siglo XX con Karinthy y su teoría de los seis grados de separación, cuya formulación fue posteriormente recogida, ampliada y puesta al día por Watts (2003), quien subrayó cómo cualquier habitante de nuestro planeta se encuentra conectado a través de no más de cinco intermediarios o seis eslabones y de qué manera el número de individuos con quienes se relaciona una persona se incrementa conforme aumenta dicha cantidad de eslabones en la cadena. 
Aunque a finales del siglo XX ya aparecen algunos ejemplos significativos de redes sociales online (con Classmate.com, 1995; AsianAvenue.com, 1997; y Sixdegrees. com, 1997, a la cabeza), no será hasta el comienzo del siglo XXI cuando se asista a su expansión y consolidación entre la comunidad internauta a partir de los ejemplos de Ryze.com (2001), LinkedIn (2002), Tribe (2002), MySpace (2003), Xing (2003, inicialmente bajo el nombre OpenBC), Orkut (2004), Flickr (2004), Facebook (2004), YouTube (2005), Twitter (2006), Pinterest (2010) o Instagram (2010), entre otros particularmente significativos.

Esa particular interacción entre prosumidores y redes sociales ha sido profusamente analizada en la literatura científica desde muy diversas perspectivas. Las redes sociales ya forman parte de la vida cotidiana de un relevante conjunto de ciudadanos que cada vez están más habituados al manejo de toda clase de dispositivos de carácter tecnológico que inciden sobre su devenir personal y profesional (Bellman, 2001), afectan profundamente a la industria de los medios al otorgar a sus usuarios unas posibilidades de interactuación y generación de contenidos propios hasta entonces desconocidas (Haythornthwaite, 2005) y contribuyen de forma decisiva a la formulación de un peculiar escenario de vínculos entre los individuos en el medio y largo plazo (Liben-Nowell y Kleinberg, 2007).

Las redes sociales conforman «un conjunto de lazos entre diversos actores (personas físicas u organizaciones), todos del mismo o similar tipo, que cuenta con un objetivo y unas normas" (Martos, 2010, p. 10), son "un servicio de Internet que permite a cualquier cibernauta construirse un perfil -público o semipúblico- dentro de un sistema gestionado por un tercero" (Bernal y Angulo, 2012, p. 25) y en todas ellas existen determinados aspectos de coincidencia, entre los que destacan el uso de internet como plataforma de difusión de contenidos, el registro previo y la creación de un perfil para poder hacer uso del servicio, la interacción con otros usuarios, la generación de información que se comparte y la creación de grupos de personas con determinados vínculos específicos (Parra y Martínez Arias, 2018).

A efectos prácticos, todo ello tiene como consecuencia un conjunto de nuevos procesos de socialización, cambios en la manera de interactuar corporaciones y audiencias o la inauguración de un espacio inédito de carácter virtual, con potencial impacto sobre la transmisión de normas, valores, actitudes y comportamientos personales y sociales (García Galera y Del Hoyo, 2013, p. 114), además de la entrada en un entorno singular donde se perciben unas muy distintas pautas de consumo por parte de los usuarios, entre las que adquieren creciente peso específico la colaboración o la búsqueda de respuestas que va mucho más allá de la tradicional actitud pasiva de los usuarios ante el poder de la industria de los medios de comunicación (Islas, 2008, p. 37).

La relación entre redes sociales e industria de la información ha sido profusamente analizada a lo largo de estos últimos años, destacando a escala internacional las aportaciones de autores como Goode (2009), Newman (2009, Harper (2010), Hermida, Fletcher, Korell y Logan (2012), Anderson (2013), Choi (2016), Fu (2016), Clark y Marchi (2017), Thurman (2018) o Wihbey, Joseph y Lazer (2019), entre otros.

En lo que atañe a la aplicación de las redes sociales al caso concreto de los cibermedios españoles, la literatura científica ha examinado de manera particularmente intensa su empleo, tanto corporativo como por parte de los profesionales de la información integrados en sus respectivas redacciones periodísticas. Flores subraya la necesidad que tienen las empresas informativas de "distribuir sus contenidos a través de estas plataformas con el fin de beneficiarse de valores añadidos como participación 
masiva e inteligencia colectiva" (2009, p. 78) mientras que Noguera enfatiza el hecho de que suponen una oportunidad estratégica de primer nivel para «llegar a determinados segmentos de audiencia que hasta el momento resultan esquivos a la industria clásica de la información» (2010, p. 179).

Por su parte Bernal pone de relieve que "con la opción de compartir sus informaciones en las redes sociales, los medios obtienen una nueva plataforma de distribución de sus contenidos, produciendo un efecto cadena entre los miembros de la red social y mejorando su repercusión» (2010, p. 28) y Martínez Fernández, Juanatey, Crespo y Mahuad concluyen que «no existe un comportamiento único ni homogéneo entre los internautas usuarios de redes sociales que consumen contenidos informativos» (2015, p. 49).

Campos, Rúas, López García y Martínez Fernández centran su análisis en el papel que los metadatos adquieren para los responsables de los medios de comunicación a la hora de alimentar redes de valor en un entorno cada vez más interconectado (2016, p. 450) al tiempo que Peñafiel opta por examinar su impacto como herramientas que facilitan el surgimiento y consolidación de narrativas transmedia bien diferentes a las propuestas del relato informativo clásico (2016, p. 172).

Pérez Soler (2018) remarca que «la relación entre medios y redes se define como frenemy ya que se basa en una colaboración competitiva y cambiante» (2018, p. 10) y Enguix alude a cinco principales efectos potenciales sobre la industria periodística que abarcan aspectos como la creación de comunidad, la difusión de contenidos, el filtro de contenidos y censura, la jerarquización de contenidos vía algoritmos y la aportación económica a su cuenta de resultados (2019, p. 165).

Pese a ello, hemos detectado la existencia de un notable vacío en las investigaciones centradas exclusivamente en precisar cuál es el peso específico exacto que las redes sociales alcanzan como herramientas generadoras de tráfico web hacia los portales de los cibermedios españoles en general y de los nativos digitales en particular.

Por consiguiente este artículo se plantea como objetivo fundamental determinar cuál es el auténtico volumen del número de usuarios que acceden a los contenidos generados por los ciber periódicos nativos digitales españoles empleando cualquiera de las diversas redes sociales en que dichos cibermedios se encuentran presentes, así como delimitar porcentualmente el alcance de cada una de estas plataformas en sus correspondientes estructuras de producción periodística.

De manera adicional, pretendemos comparar la relevancia cuantitativa de las redes sociales en relación con los otros dos habituales cauces de acceso a los contenidos informativos de los cibermedios, el tráfico directo y el procedente de motores de búsqueda vía posicionamiento SEO, así como establecer un potencial escenario de futuro a corto, medio y largo plazo.

Nuestra hipótesis es que hoy en día se otorga a las redes sociales una relevancia cuantitativa en su capacidad para generar tráfico web que excede de sus verdaderas posibilidades. Tal convicción se percibe en la opinión pública, en los foros empresariales que periódicamente son organizados por representantes de la industria de la información, en los profesionales del periodismo, en los congresos científicos que reúnen a expertos en la docencia e investigación académica de la información periodística digital y en la producción bibliográfica por parte de dichos especialistas en la materia.

Como consecuencia de ello, los cibermedios realizan un considerable esfuerzo para participar en el flujo de contenidos generados por parte de las redes sociales que a efectos prácticos se concreta en su presencia corporativa y en la de los profesionales que forman parte de su plantilla pero cuya verdadera justificación estratégica resulta dudosa. 
Consideramos que este sobredimensionamiento tiene que ver con los elevados niveles de penetración de las redes sociales entre la ciudadanía en general y entre los componentes de la industria de la información en particular, así como con la situación de reconversión tecnológica, epistemológica y ética que hoy en día vive el negocio del periodismo con factores de transformación como el paso de un mercado de demanda local a otro de oferta global, el cambio del paradigma en el acceso y difusión del conocimiento, la pérdida del monopolio en la producción y distribución de los contenidos o la actitud crecientemente crítica de las audiencias que deviene en un escenario global de deterioro de su credibilidad, erosión de su imagen de marca y dramática merma de sus márgenes comerciales de rentabilidad.

\section{Metodología utilizada}

Con el fin de llevar a cabo el análisis del impacto que las redes sociales tienen en el conjunto del tráfico web generado por los cibermedios nativos digitales españoles se ha procedido en primer lugar a la selección de una muestra que resulte científicamente significativa. Para ello hemos empleado el criterio cuantitativo del número mensual de usuarios únicos que acceden a sus respectivos portales online, empleando dos diferentes métricas de referencia aportadas por las empresas comScore y OJD Interactiva que constituyen sendos patrones de medición en la industria española de la información.

En el caso de comScore se trata de una herramienta de tipo user centric que no sólo proporciona información sobre los visitantes a la página web sino que además reporta acerca de los sitios por los que navegan los usuarios antes y después, la audiencia compartida con los potenciales competidores e incluso la evolución en el tráfico web de estos últimos.

Este sistema se basa en la combinación de datos censales y muestrales. Los primeros se obtienen a partir de la herramienta propietaria de analítica web DAX (Digital Analytix), cuyo funcionamiento se sustenta en la tecnología híbrida UDM (Unified Digital Measurement), que recibe el tráfico censal reenviado a través de etiquetas y descarta así, con algunas limitaciones, el tráfico no humano. Una vez obtenido el cociente de CPP (Cookies per Person) realiza una extrapolación de cuántos individuos representan el número total de cookies generadas.

En lo que se refiere a los datos muestrales proceden de un grupo de más de 31.000 usuarios, que han accedido a que les sea instalado en sus dispositivos de acceso a los contenidos web (fundamentalmente ordenadores personales y teléfonos móviles) el equivalente a un audímetro que incorpora un mini programa informático o cProxy. Este software permite registrar todos los sitios web a los que ha accedido el usuario.

Por su parte la metodología de la Oficina de Justificación de la Difusión aplicada al entorno online u OJD Interactiva se adecúa a la normativa de la International Federation of Audit Bureaux of Certification (IFABC), organización que agrupa a entidades de certificación que acreditan la verificación y el control de la difusión de las publicaciones pertenecientes a 36 países.

La propuesta de OJD Interactiva contempla una dualidad de normativas técnicas de control de accesos tanto desde equipos informáticos conectados de manera permanente a una infraestructura online como desde dispositivos en movimiento como tabletas electrónicas y teléfonos móviles, por lo que se aproxima en mayor medida a 
los sistemas de medición web centric. Dicha metodología es la que explica que, por lo general, sus datos de difusión sean más elevados que los obtenidos mediante el sistema de análisis de comscore.

Podemos ver las cifras de ambas metodologías de medición en las dos siguientes tablas, la primera corresponde al sistema empleado por comScore y la segunda al utilizado por OJD Interactiva.

Tabla 1. Número de usuarios únicos mensuales durante diciembre de 2019

\begin{tabular}{|l|l|l|}
\hline Nombre del cibermedio & URL & $\begin{array}{l}\text { Número de usuarios únicos } \\
\text { mensuales }\end{array}$ \\
\hline El Español & https://www.elespanol.com/ & 17.019 .000 \\
\hline El Confidencial & https://www.elconfidencial.com/ & 15.218 .000 \\
\hline OK Diario & https://okdiario.com/ & 11.896 .000 \\
\hline El Diario & https://www.eldiario.es/ & 9.188 .000 \\
\hline Huffington Post & https://www.huffingtonpost.es/ & 7.839 .000 \\
\hline Periodista Digital & https://www.periodistadigital.com/ & 6.755 .000 \\
\hline Voz Pópuli & https://www.vozpopuli.com/ & 5.828 .000 \\
\hline Libertad Digital & https://www.libertaddigital.com/ & 5.538 .000 \\
\hline Público & https://www.publico.es/ & 5.360 .000 \\
\hline El Independiente & https://www.elindependiente.com/ & $\begin{array}{l}\text { No proporciona cifras desde } \\
\text { julio de 2019 }\end{array}$ \\
\hline
\end{tabular}

Fuente: comscore

Tabla 2. Número de usuarios únicos mensuales durante diciembre de 2019

\begin{tabular}{|l|l|l|}
\hline Nombre del cibermedio & URL & $\begin{array}{l}\text { Número de usuarios únicos } \\
\text { mensuales }\end{array}$ \\
\hline El Español & https://www.elespanol.com/ & 51.517 .000 \\
\hline El Confidencial & https://www.elconfidencial.com/ & No auditado \\
\hline OK Diario & https://okdiario.com/ & No auditado \\
\hline El Diario & https://www.eldiario.es/ & 15.583 .000 \\
\hline Huffington Post & https://www.huffingtonpost.es/ & No auditado \\
\hline Periodista Digital & https://www.periodistadigital.com/ & No auditado \\
\hline Voz Pópuli & https://www.vozpopuli.com/ & No auditado \\
\hline Libertad Digital & https://www.libertaddigital.com/ & No auditado \\
\hline Público & https://www.publico.es/ & 12.676 .000 \\
\hline El Independiente & https://www.elindependiente.com/ & 3.807 .000 \\
\hline
\end{tabular}

Fuente: OJD Interactiva 
Más allá de las discrepancias puntuales entre ambos sistemas y del hecho de que no todos los cibermedios se encuentren auditados en ambos entornos, la combinación de las dos métricas nos permite concluir que los diez nativos digitales españoles que a fecha de 31 de diciembre de 2019 cuentan con un mayor número de visitantes únicos en sus portales web son: El Español, El Confidencial, OK Diario, El Diario, Huffington Post, Periodista Digital, Voz Pópuli, Libertad Digital, Público y El Independiente.

A continuación se ha procedido al examen de estos diez portales web, empleando la herramienta de análisis SimilarWeb. Se trata de un software que permite examinar el tráfico web de un portal online proporcionando datos sobre facetas como clasificación del sitio en función de las visitas totales recibidas a lo largo de un semestre, páginas vistas en cada visita y duración promedio de la misma, sitios de referencia y de destino, perfil del visitante o fuentes de tráfico, entre otras muchas.

La elección de esta herramienta responde al cumplimiento de los requisitos de acreditada robustez, notable flexibilidad en su configuración y capacidad contrastada para la generación de datos fiables. Además, SimilarWeb ha sido empleada con eficacia en distintas investigaciones planteadas en el campo de las ciencias sociales en general y de la comunicación y el periodismo en particular. En lo que se refiere al ámbito del ciberperiodismo destacan las aportaciones realizadas por autores como Bosio (2017), Thorsen (2017), Austin (2019) o Schapals, Maeres y Hanusch (2019) a nivel internacional, así como las de Rodríguez Martínez, Codina y Pedraza (2010), Peña, Lazkano y García González (2016), Rojas y Marín (2016), Ufarte y Murcia (2018) o Pujol (2019) en el caso de España.

A partir de la herramienta se elaboraron cuatro diferentes tablas. La primera de ellas proporciona un conjunto de datos generales de tráfico web para cada cibermedio, distinguiéndose los siguientes apartados: procedencia del tráfico por países (considerándose los cinco principales); tasa de rebote (entendida en su faceta formal de no realizar ninguna clase de interacción en la página visitada más allá de la lectura de su contenido); y duración media de las visitas de sus usuarios (expresada en minutos y segundos).

La segunda tabla muestra lo que supone el porcentaje del tráfico generado desde las redes sociales sobre el tráfico web total de cada uno de los cibermedios nativos digitales.

La tercera tabla alude a la distribución del tráfico generado desde las redes sociales en función de la diferente tipología de las mismas (considerándose las cinco principales en cada caso).

Finalmente la cuarta tabla establece una comparación entre el tráfico web generado desde las redes sociales y el que se aporta mediante los procedimientos de generación de tráfico directo y a través del empleo de motores de búsqueda vía posicionamiento SEO.

La medición de los portales web de los diez cibermedios nativos digitales se inició el 20 de enero y concluyó el 15 de febrero de 2020.

\section{Resultados y discusión}

En esta tabla se proporcionan los datos generales correspondientes a la procedencia del tráfico web generado por los visitantes únicos de los cinco países más relevantes en cada caso, la tasa de rebote o bounce rate entendida en los términos 
apuntados con anterioridad de no realizar ninguna clase de interacción en la página visitada más allá de la potencial lectura de su contenido y la duración media de la visita del usuario promedio expresada en minutos y segundos.

Lógicamente en todos los ciber periódicos los usuarios procedentes de España constituyen la gran mayoría de los visitantes a los portales web de los nativos digitales. No obstante debe señalarse el particular caso de El Español donde únicamente se alcanza un índice del 46,85\% frente a una media superior al 80\% del resto de sus competidores.

La tasa de rebote marca la existencia de tres grupos distintos de cibermedios: los que superan el 60\%, con los ejemplos de El Español, OK Diario y, de manera especial, El Independiente donde tal situación afecta a más de dos tercios de sus visitantes únicos; el tramo mayoritario de los que se sitúan entre el 50\% y el 60\%, con Huffington Post, El Confidencial, El Diario y Voz Pópuli; y quienes se encuentran por debajo de la barrera de la mitad de los usuarios únicos, con Periodista Digital, Público y Libertad Digital.

En cuanto a la duración media de la visita de un internauta promedio se detectan remarcables diferencias entre los cibermedios analizados. Huffington Post y El Independiente son quienes muestran una menor capacidad para retener a sus visitas (apenas dos minutos) mientras que en el extremo contrario se sitúan Público, Libertad Digital y Voz Pópuli (todos por encima de los seis minutos).

Tabla 3. Datos generales de tráfico web

\begin{tabular}{|c|c|c|c|}
\hline $\begin{array}{l}\text { Nombre del } \\
\text { cibermedio }\end{array}$ & $\begin{array}{l}\text { Procedencia por países } \\
\text { ( } 5 \text { principales) }\end{array}$ & Tasa de rebote & $\begin{array}{l}\text { Duración media de la } \\
\text { visita de un usuario } \\
\text { tipo }\end{array}$ \\
\hline El Español & $\begin{array}{l}\text { - España: } 46,85 \% \\
\text { - Argentina: } 10,90 \% \\
\text { - México: } 10,63 \% \\
\text { - Colombia: } 6,91 \% \\
\text { - Chile: } 4,87 \%\end{array}$ & $61,34 \%$ & 2 minutos 50 segundos \\
\hline El Confidencial & $\begin{array}{l}\text { - España: } 75,41 \% \\
\text { - México: } 3,52 \% \\
\text { - Argentina: 3,29\% } \\
\text { - Estados Unidos: 2,13\% } \\
\text { - Colombia: } 2,05 \%\end{array}$ & $51,42 \%$ & 4 minutos 20 segundos \\
\hline OK Diario & $\begin{array}{l}\text { - España: } 67,21 \% \\
\text { - México: } 6,95 \% \\
\text { - Argentina: } 3,93 \% \\
\text { - Colombia: } 3,67 \% \\
\text { - Venezuela: } 2,55 \%\end{array}$ & $62,42 \%$ & 3 minutos 38 segundos \\
\hline El Diario & $\begin{array}{l}\text { - España: } 83,31 \% \\
\text { - Estados Unidos: } 2,27 \% \\
\text { - México: } 1,52 \% \\
\text { - Reino Unido: } 1,34 \% \\
\text { - Argentina: } 1,21 \%\end{array}$ & $56,38 \%$ & 5 minutos 6 segundos \\
\hline Huffington Post & $\begin{array}{l}\text { - España: } 81,99 \% \\
\text { - México: 2,94\% } \\
\text { - Estados Unidos: 2,00\% } \\
\text { - Argentina: } 1,82 \% \\
\text { - Colombia: } 1,36 \%\end{array}$ & $50,72 \%$ & 1 minuto 24 segundos \\
\hline
\end{tabular}




\begin{tabular}{|c|c|c|c|}
\hline $\begin{array}{l}\text { Nombre del } \\
\text { cibermedio }\end{array}$ & $\begin{array}{l}\text { Procedencia por países } \\
\text { ( } 5 \text { principales) }\end{array}$ & Tasa de rebote & $\begin{array}{l}\text { Duración media de la } \\
\text { visita de un usuario } \\
\text { tipo }\end{array}$ \\
\hline Periodista Digital & $\begin{array}{l}\text { - España: } 82,51 \% \\
\text { - Argentina: } 3,15 \% \\
\text { - México: } 2,04 \% \\
\text { - Estados Unidos: } 1,97 \% \\
\text { - Colombia: } 1,12 \%\end{array}$ & $45,18 \%$ & 3 minutos 22 segundos \\
\hline Voz Pópuli & $\begin{array}{l}\text { - España: } 85,71 \% \\
\text { - Estados Unidos: 2,03\% } \\
\text { - México: } 1,47 \% \\
\text { - Argentina: } 1,35 \% \\
\text { - Reino Unido: } 1,25 \%\end{array}$ & $57,38 \%$ & 6 minutos 23 segundos \\
\hline Libertad Digital & $\begin{array}{l}\text { - España: } 86,82 \% \\
\text { - Estados Unidos: } 2,61 \% \\
\text { - México: } 1,52 \% \\
\text { - Argentina: } 1.10 \% \\
\text { - Colombia: } 0,93 \%\end{array}$ & $49,57 \%$ & 6 minutos 16 segundos \\
\hline Público & $\begin{array}{l}\text { - España: } 81,81 \% \\
\text { - Estados Unidos: } 2,88 \% \\
\text { - México: } 1,73 \% \\
\text { - Argentina: } 1,57 \% \\
\text { - Colombia: } 1,11 \%\end{array}$ & $48,08 \%$ & 6 minutos 0 segundos \\
\hline El Independiente & $\begin{array}{l}\text { - España: } 78,85 \% \\
\text { - Italia: } 4,57 \% \\
\text { - Colombia: } 2,28 \% \\
\text { - México: } 2,26 \% \\
\text { - Estados Unidos: } 2,01 \%\end{array}$ & $67,65 \%$ & 2 minutos 19 segundos \\
\hline
\end{tabular}

Fuente: Elaboración propia a partir de la herramienta SimilarWeb

En la cuarta tabla se muestra el peso específico que las redes sociales, consideradas en su conjunto, adquieren sobre el total del tráfico web que es generado por los diferentes nativos digitales españoles examinados. Como punto de partida, llama la atención las grandes diferencias que se perciben entre unos y otros cibermedios: frente al muy escaso porcentaje que suponen para Libertad Digital se encuentra el caso de Huffington Post que prácticamente quintuplica dicha cifra.

Pese a ello, en todos los casos las redes sociales se sitúan por debajo del listón estratégico del $20 \%$ en cuanto a su capacidad de generación de tráfico web se refiere, distinguiéndose cuatro grupos (considerados en tramos de cinco en cinco puntos porcentuales): influencia inferior al 5\%, con la única excepción que supone Libertad Digital; impacto situado entre el 5 y el 10\% (el tramo mayoritario), con OK Diario, El Español, El Confidencial, El Independiente, Voz Pópuli y Periodista Digital; del 11 al 15\%, donde se incluye El Diario; y más del 15\%, con Público y Huffington Post.

Si se establece una comparación con los valores hallados en la tabla anterior (número tres) no se detecta ninguna relación significativa entre el porcentaje del tráfico generado desde las redes sociales y magnitudes como la procedencia del tráfico web generado por países, la tasa de rebote o la duración media de la visita del usuario promedio. 
DAVID PARRA VALCARCE Y CHARO ONIEVA MALLERO

ANÁLISIS DEL IMPACTO DE LAS REDES SOCIALES SOBRE EL TRÁFICO

WEB DE LOS CIBERMEDIOS NATIVOS DIGITALES ESPAÑOLES

Tabla 4. Porcentaje del tráfico generado desde las redes sociales sobre el tráfico web total

\begin{tabular}{|l|l|}
\hline Nombre del cibermedio & $\begin{array}{l}\text { Porcentaje del tráfico generado } \\
\text { por las RRSS }\end{array}$ \\
\hline El Español & $5,58 \%$ \\
\hline El Confidencial & $6,84 \%$ \\
\hline OK Diario & $5,37 \%$ \\
\hline El Diario & $12,99 \%$ \\
\hline Huffington Post & $18,42 \%$ \\
\hline Periodista Digital & $8,56 \%$ \\
\hline Voz Pópuli & $7,41 \%$ \\
\hline Libertad Digital & $3,70 \%$ \\
\hline Público & $15,67 \%$ \\
\hline El Independiente & $7,03 \%$ \\
\hline
\end{tabular}

Fuente: Elaboración propia a partir de la herramienta SimilarWeb

En el gráfico número uno se reflejan los mismos datos de la tabla número cuatro con el objetivo fundamental de proporcionar una visión global más clara del conjunto de cifras expresadas con anterioridad.

Gráfico 1. Porcentaje del tráfico generado desde las redes sociales sobre el tráfico web total

\section{Porcentaje del tráfico generado por las RRSS}

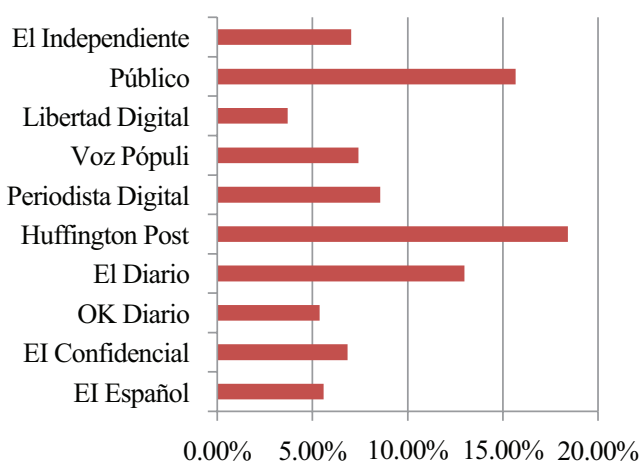

Porcentaje del tráfico generado por las RRSS

Fuente: Elaboración propia a partir de la herramienta SimilarWeb

La tabla número cinco centra su atención en el desglose del tráfico procedente de las redes sociales en función de las principales plataformas en las que se encuentran presentes los distintos cibermedios estudiados.

Los datos permiten advertir tres cuestiones significativas. La primera de ellas tiene que ver con el hecho de que Facebook es, con mucho, la red social más relevante (es el caso de siete de los diez cibermedios aquí tenidos en cuenta), con un 
porcentaje sobre el conjunto de las redes sociales superior a la mitad y con los casos particulares de Huffington Post y Público, en los que supera el umbral de los dos tercios.

Una segunda consideración tiene que ver con que los tres cibermedios en los que Twitter es la principal red social en cuanto a grado de impacto se refiere (Voz Pópuli, Libertad Digital y El Independiente) se encuentran entre los cuatro últimos (la única excepción sería Público) según el número de visitantes únicos mensuales, tal y como puede advertirse en los datos proporcionados por comscore y recogidos en la tabla número uno.

Y, por último, en todos los casos YouTube se convierte en la tercera opción para los cibermedios que han sido examinados, con porcentajes que van desde el 2,34\% para Público hasta el 14,70\% en el caso de Libertad Digital. Las restantes redes sociales (Instagram, LinkedIn, Pinterest, Whatsapp...) contribuyen de manera ínfima a la generación de tráfico web, situándose siempre por debajo del 3\%.

Tabla 5. Distribución del tráfico web generado desde las redes sociales

\begin{tabular}{|c|c|}
\hline Nombre del cibermedio & $\begin{array}{l}\text { Distribución del tráfico por RRSS } \\
\text { ( } 5 \text { principales) }\end{array}$ \\
\hline El Español & $\begin{array}{l}\text { - Facebook: } 55,32 \% \\
\text { - Twitter: } 29,21 \% \\
\text { - YouTube: } 12,13 \% \\
\text { - Whatsapp: } 0,81 \% \\
\text { - LinkedIn: } 0,66 \%\end{array}$ \\
\hline El Confidencial & $\begin{array}{l}\text { - Facebook: } 57,22 \% \\
\text { - Twitter: } 32,87 \% \\
\text { - YouTube: } 5,19 \% \\
\text { - LinkedIn: } 2,73 \% \\
\text { - Whatsapp: } 1,05 \%\end{array}$ \\
\hline OK Diario & $\begin{array}{l}\text { - Facebook: } 59,09 \% \\
\text { - Twitter: } 29,28 \% \\
\text { - YouTube: } 8,85 \% \\
\text { - Pinterest: } 0,74 \% \\
\text { - Whatsapp: } 0,58 \%\end{array}$ \\
\hline El Diario & $\begin{array}{l}\text { - Facebook: } 51,10 \% \\
\text { - Twitter: } 43,46 \% \\
\text { - YouTube: } 3,56 \% \\
\text { - Whatsapp: } 0,79 \% \\
\text { - LinkedIn: } 0,36 \%\end{array}$ \\
\hline Huffington Post & $\begin{array}{l}\text { - Facebook: } 68,10 \% \\
\text { - Twitter: } 28,33 \% \\
\text { - YouTube: } 2,29 \% \\
\text { - Pinterest: } 0,81 \% \\
\text { - Instagram: } 0,15 \%\end{array}$ \\
\hline Periodista Digital & $\begin{array}{l}\text { - Facebook: } 58,35 \% \\
\text { - Twitter: } 34,04 \% \\
\text { - YouTube: } 5,95 \% \\
\text { - Whatsapp: } 0,63 \% \\
\text { - DailyMotion: } 0,52 \%\end{array}$ \\
\hline
\end{tabular}




\begin{tabular}{|c|c|}
\hline Nombre del cibermedio & $\begin{array}{l}\text { Distribución del tráfico por RRSS } \\
\text { ( } 5 \text { principales) }\end{array}$ \\
\hline Voz Pópuli & $\begin{array}{l}\text { - Twitter: } 58,85 \% \\
\text { - Facebook: } 29,22 \% \\
\text { - YouTube: } 7,53 \% \\
\text { - Whatsapp: } 2,32 \% \\
\text { - LinkedIn: } 1,03 \%\end{array}$ \\
\hline Libertad Digital & $\begin{array}{l}\text { - Twitter: } 44,34 \% \\
\text { - Facebook: } 37,72 \% \\
\text { - YouTube: } 14,70 \% \\
\text { - Whatsapp: } 1,69 \% \\
\text { - Pinterest: } 0,36 \%\end{array}$ \\
\hline Público & $\begin{array}{l}\text { - Facebook: } 71,52 \% \\
\text { - Twitter: } 24,95 \% \\
\text { - YouTube: } 2,34 \% \\
\text { - Whatsapp: } 0,45 \% \\
\text { - Reddit: } 0,26 \%\end{array}$ \\
\hline El Independiente & $\begin{array}{l}\text { - Twitter: } 47,96 \% \\
\text { - Facebook: } 39,19 \% \\
\text { - YouTube: } 9,36 \% \\
\text { - LinkedIn: } 2,31 \% \\
\text { - Whatsapp: } 0,70 \%\end{array}$ \\
\hline
\end{tabular}

Fuente: Elaboración propia a partir de la herramienta SimilarWeb

La tabla número seis permite realizar una comparación entre el volumen de tráfico web generado a través de las redes sociales y el que es aportado mediante el tráfico directo y a través del empleo de motores de búsqueda vía estrategias de posicionamiento SEO.

Tal y como ya se anticipa en los datos englobados en la tabla número cuatro y explicitados con posterioridad en el gráfico número uno, las redes sociales son siempre un elemento menor en comparación con las dos restantes variables sin que exista ninguna excepción a esta regla general.

Huffington Post constituye el ciberperiódico en el que esta diferencia es menos significativa $(18,42 \%$ frente a $28,86 \%$ y $22,84 \%)$ si bien debe matizarse que este medio posee una estructura de reparto del tráfico web distinta a los otros nueve examinados dado el notable peso específico que adquiere en su estructura productiva el tráfico web generado por otras páginas o enlaces (en términos técnicos, referrals), un aspecto insignificante para los restantes y que en su caso se sitúa por encima del 27\% (con un medio como la versión digital de El País que supone alrededor de tres cuartos del total de dicho tráfico).

Tabla 6. Comparación del tráfico web generado por las RRSS y el procedente de tráfico directo y buscadores

\begin{tabular}{|l|l|l|l|}
\hline Nombre del cibermedio & Tráfico de redes sociales & Tráfico directo & Tráfico vía buscadores \\
\hline El Español & $5,58 \%$ & $32,57 \%$ & $59,21 \%$ \\
\hline El Confidencial & $6,84 \%$ & $52,05 \%$ & $35,61 \%$ \\
\hline OK Diario & $5,37 \%$ & $40,08 \%$ & $52,83 \%$ \\
\hline El Diario & $12,99 \%$ & $58,38 \%$ & $25,57 \%$ \\
\hline
\end{tabular}


DAVID PARRA VALCARCE Y CHARO ONIEVA MALLERO

ANÁLISIS DEL IMPACTO DE LAS REDES SOCIALES SOBRE EL TRÁFICO

WEB DE LOS CIBERMEDIOS NATIVOS DIGITALES ESPAÑOLES

\begin{tabular}{|l|l|l|l|}
\hline Nombre del cibermedio & Tráfico de redes sociales & Tráfico directo & Tráfico vía buscadores \\
\hline Huffington Post & $18,42 \%$ & $28,86 \%$ & $22,84 \%$ \\
\hline Periodista Digital & $8,56 \%$ & $58,86 \%$ & $29,78 \%$ \\
\hline Voz Pópuli & $7,41 \%$ & $54,57 \%$ & $29,72 \%$ \\
\hline Libertad Digital & $3,70 \%$ & $68,37 \%$ & $24,67 \%$ \\
\hline Público & $15,67 \%$ & $56,93 \%$ & $21,89 \%$ \\
\hline El Independiente & $7,03 \%$ & $38,95 \%$ & $45,17 \%$ \\
\hline
\end{tabular}

Fuente: Elaboración propia a partir de la herramienta SimilarWeb

Este gráfico número dos explícita los datos proporcionados en la anterior tabla. Además, permite advertir de una manera más clara las diferencias existentes entre los cibermedios cuando se comparan de manera individual la magnitud redes sociales con las de tráfico directo y buscadores.

Si la comparación se establece con la magnitud de tráfico directo, la ratio iría desde el máximo 0,638 para Huffington Post hasta el mínimo 0,054 alcanzado por Libertad Digital.

En el caso de que el cotejo se lleve a cabo con el tráfico generado mediante el uso de buscadores, nos encontramos con dos cibermedios cuyo cociente resulta muy superior a los restantes, Huffington Post $(0,806)$ y Público $(0,715)$, cuya situación contrasta con la de El Español $(0,094)$ y OK Diario $(0,101)$.

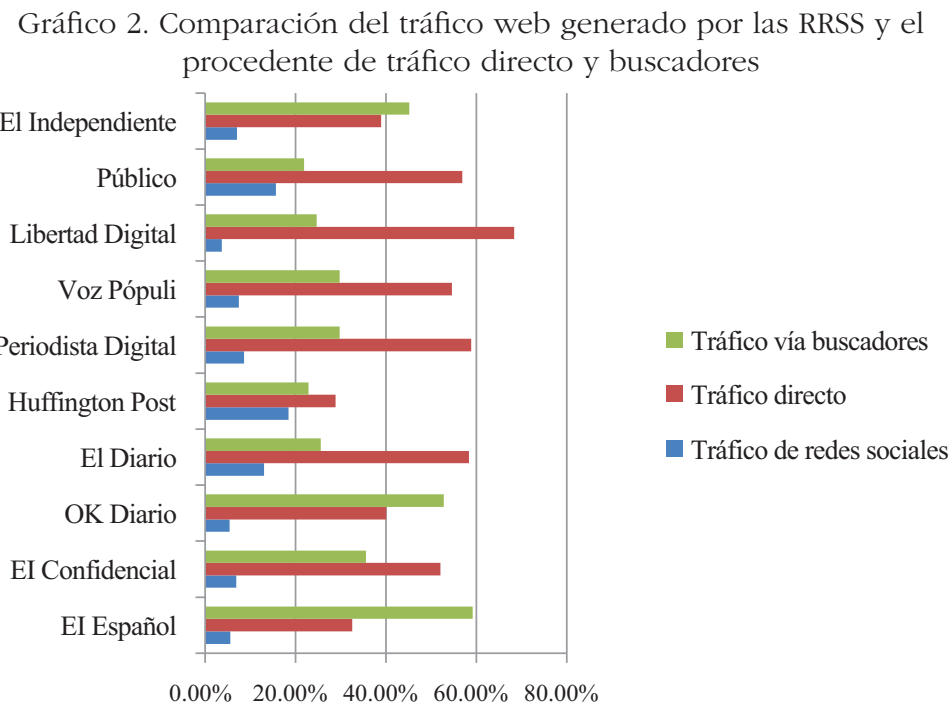

Fuente: Elaboración propia a partir de la herramienta SimilarWeb

\section{Conclusiones}

En términos generales el peso específico porcentual que las redes sociales tienen como elemento estratégico de generación de tráfico web en los cibermedios nativos 
digitales españoles se sitúa en todos los casos considerados por debajo del umbral del 20\% sobre el tráfico total. Estos datos contrastan con la relevancia que se otorga a esta clase de plataformas como herramientas de generación de tráfico web y que es percibida tanto en la opinión pública en general como en foros empresariales y científicos y en la producción bibliográfica generada por los investigadores y docentes especializados en el ámbito del periodismo digital, así como en el propio esfuerzo que es llevado a cabo por parte de las empresas periodísticas fomentando su activa participación en las mismas.

En los diez casos examinados se ha detectado, verificado y contrastado que las redes sociales tienen menor impacto en los procesos de generación de tráfico web a los portales de los cibermedios nativos digitales españoles que opciones como el tráfico directo o la búsqueda de contenidos a través de motores guiados por procedimientos de posicionamiento SEO.

La relevancia cuantitativa que las redes sociales tienen como elemento estratégico de generación de tráfico web en los cibermedios nativos digitales españoles es muy distinta según los casos, abarcando un amplio rango desde un mínimo del 3,70\% (Libertad Digital) hasta un máximo del 18,42\% (Huffington Post).

Facebook es la red social más relevante para la gran mayoría de los nativos digitales (siete de los diez examinados), con un porcentaje sobre el conjunto de las mismas superior a la mitad y con los casos particulares de Huffington Post y Público en los que supera el umbral de dos tercios. Twitter es la siguiente red social en cuanto a grado de impacto, convirtiéndose en la principal fuente para tres de los cibermedios (Voz Pópuli, Libertad Digital y El Independiente), que coinciden con tres de los cuatro que cuentan con un menor número de visitantes únicos mensuales de los diez aquí considerados.

En ninguno de los casos estudiados se detectaron interrelaciones estadísticamente significativas entre el peso específico de las redes sociales en el tráfico web total generado y variables como la procedencia de dicho tráfico por países, la tasa de rebote o la duración media de las visitas de sus usuarios.

\section{Bibliografía}

Andersen, P. (2007). What is Web 2.0? Ideas, technologies and implications for education. Bristol, Reino Unido: JISC.

Anderson, C.W. (2013). Rebuilding the News: Metropolitan Journalism in the Digital Age. Filadelfia, Estados Unidos: Temple University.

Austin, L. (2019). Five trends to watch in Journalism. Studia Universitatis Babes-Bolyai Ephemerides, 64(1), pp. 45-66. Recuperado de https://www.ceeol.com/search/article-detail?id=803450

Bellman, B. (2001). Computer Networks as Social Networks. Science, 293 (5537), pp. 2031-2034. doi: https://doi.org/10.1126/science.1065547

Bernal, A.I. (2010). Presencia de las redes sociales en los cibermedios españoles. Textual $\varepsilon$ Visual Media, 3, pp. 25-42. Recuperado de http://textualvisualmedia.com/index.php/ txtvmedia/article/view/40/32

Bird, S.E. (2011). Are We All Producers Now? Convergence and Media Audience Practices. Cultural Studies, 25(4-5), pp. 502-516. doi: https://doi.org/10.1080/09502386.2011.600532

Bofil, L. (2013). Constructivism and Collaborating Using Web 2.0 Technology. Journal of Applied Learning Technology, 3(2), pp. 31-37. Recuperado de https://www.academia.edu/3840497/ Constructivism_and_Collaboration_Using_Web_2.0_Technology

Bosio, D. (2017). Journalism and Social Media. Londres, Reino Unido: Palgrave Macmillan. 
Boyd, D. y Ellison, N. (2007). Social Network Sites: Definition, History and Scholarship. Journal of Computer-mediated Communication, 13(1), pp. 210-230. doi: https://doi.org/10.1111/j.10836101.2007.00393.x

Briggs, M. (2007). Periodismo 2.0. Austin, Estados Unidos: Knight Foundation.

Bruns, A. (2009). From Prosumer to Produser: Understanding User-Led Content Creation. Transforming Audiences Conference. Londres (3-4 septiembre), pp. 1-18. Recuperado de https://documents. $\mathrm{pub} /$ document/from-prosumer-to-produser-understanding-user-led-content-creation.html

Campos, F., Rúas, J., López García, X. y Martínez Fernández, V.A. (2016). Impacto de las redes sociales en el periodismo. El Profesional de la Información, 25(3), pp. 449-457. doi: https:// doi.org/10.3145/epi.2016.may.15

Caruso, S. (2018). Towards Understanding the Role of Web 2.0 Technology in Self-Directed Learning And Job Performance. Contemporary Issues in Education Research, 11(3), pp. 89-98. doi: https://doi.org/10.19030/cier.v11i3.10180

Cebrián, M. (2010). Desarrollo del periodismo en Internet. Sevilla, España: Comunicación Social.

Choi, J. (2016). News Internalizing and Externalizing: The Dimensions of News Sharing on Online Social Networking Sites. Journalism \& Mass Communication Quarterly, 93(4), pp. 816-835. doi: https://doi.org/10.1177/1077699016628812

Clark, L.S. y Marchi, R. (2017). Young People and the Future of News. Social Media and the Rise of Connective Journalism. Cambridge, Reino Unido: Cambridge University Press.

Coleman, D. y Levine, S. (2008). Collaboration 2.0: technology and best practices for successful collaboration in a Web 2.0 world. Silicon Valley, Estados Unidos: Happy About.

Di Nucci, D. (1999). Fragmented Future. Print Magazine, 53(4), 32, pp. 221-222. Recuperado de http://darcyd.com/fragmented_future.pdf

Enguix, S. (2019). Diez años de redes sociales y periodismo: riesgos y retos en el ecosistema informativo del siglo XXI. Dígitos, 5, pp. 164-183. doi: http://dx.doi.org/10.7203/rd.v0i5.146

Flores, J. (2009). Nuevos modelos de comunicación, perfiles y tendencias en las redes sociales. Comunicar, 33(17), pp. 73-81. doi: https://doi.org/10.3916/c33-2009-02-007

Fu, J.S. (2016). Leveraging Social Network Analysis for Research on Jornalism in the Information Age. Journal of Communication, 66(2), pp. 299-313. doi: https://doi.org/10.1111/jcom.12212

Fumero, A. y Roca, G. (2007). Web 2.0. Madrid, España: Fundación Orange.

García Avilés, J. A. y González Esteban, J. L. (2013). Cibermedios nativos españoles: explorando modelos de rentabilidad. Trípodos, 30, pp. 153-167. Recuperado de http://www.tripodos. com/index.php/Facultat_Comunicacio_Blanquerna/article/view/50/360

García Galera, M.C. y Del Hoyo, M. (2013). Redes sociales, un medio para la movilización juvenil. Zer Revista de Estudios de Comunicación, 18(34), pp. 111-125. Recuperado de https://www.ehu.eus/ojs/index.php/Zer/article/view/10649/9887

Gardner,B.(2011). ResponsiveWebDesign:EnrichingtheUserExperience.Sigma, 11(1),pp.13-19.Recuperado de https://studylib.net/doc/8225889/responsive-web-design--enriching-the-user-experience

González Esteban, J.L. (2010). Auge y caída de Soitu, un ejemplo de medio nativo digital en España. Comunicación \& Sociedad, 23(2), pp. 267-288. Recuperado de https://revistas. unav.edu/index.php/communication-and-society/article/view/36241/30672

Grosseck, G. (2009). To use or not to use Web 2.0 in higher education?. Procedia Social and Behavioural Sciences, 1(1), pp. 478-482. doi: https://doi.org/10.1016/j.sbspro.2009.01.087

Goode, L. (2009). Social news, citizen Journalism and democracy. New Media \& Society, 11(8), pp. 1287-1305. doi: https://doi.org/10.1177/1461444809341393

Harper, R. (2010). The Social Media Revolution: Exploring the Impact on Journalism and News Media Organizations. Inquiries Journal, 2(3). Recuperado de http://www.inquiriesjournal.com/articles/202/the-social-media-revolution-exploring-the-impact-on-journalism-and-news-media-organizations

Harris, A. y Rea, A. (2019). Web 2.0 and Virtual World Technologies: A Growing Impact on IS Education. Journal of Information Systems Education, 20(2), pp. 137-144. Recuperado de https://aisel.aisnet.org/cgi/viewcontent.cgi?article=1271\&context=jise 
Haythornthwaite, C. (2005). Social networks and Internet connectivity effects. Information, Communication ESociety, 8(2),pp.125-147. doi: https://doi.org/10.1080/13691180500146185

Hermida, A., Fletcher, F., Korell, D. y Logan, D. (2012). Share, Like, Recommend. Decoding the social media news consumer. Journalism Studies, 13(5-6), pp. 815-824. doi: https://doi.org/ 10.1080/1461670X.2012.664430

Hernández Soriano, F. y Cristóbal, E. (2014). Evaluación de las herramientas Web 2.0 en el periodismo digital: el caso de VilaWeb. Historia y Comunicación Social, 19(1), pp. 79-91. doi: http://dx.doi.org/10.5209/rev_HICS.2014.v19.44942

Hill, R. (2016). What an Algorithm Is. Philosophy and Technology, 29(1), pp. 35-59. doi: https:// doi.org/10.1007/s13347-014-0184-5

Iglesias, M. y González Díaz, C. (2012) Análisis de la calidad de la web del ciberdiario Vilaweb. Hipertext.net, 10. Recuperado de https://www.upf.edu/hipertextnet/numero-10/ vilaweb-analisis-calidad-web-ciberdiario.html

Islas, J.O. (2008). El prosumidor. El actor comunicativo de la sociedad de la ubicuidad. Palabra Clave, 11(1), pp. 29-39. Recuperado de https://palabraclave.unisabana.edu.co/index.php/ palabraclave/article/view/1413/1723

Kamel Boulos, M. y Wheeler, S. (2007). The emerging Web 2.0 social software: an enabling suite of sociable technologies in health and health care education. Health Information and Libraries Journal, 24, pp. 2-23. doi: https://doi.org/10.1111/j.1471-1842.2007.00701.x

Liben-Nowell, D. y Kleinberg, J. (2007). The link-prediction problem for social networks. Journal of the American Society for Information Science and Technology, 58(7), pp. 1019-1031. doi: https://doi.org/10.1002/asi.20591

Martínez Fernández, V.A., Juanatey, O., Crespo, V. y Mahauad, M. D. (2015). Prensa nativa digital y redes sociales: cibermedios en Facebook y Twitter. International Journal of Information Systems and Software Engineering for Big Companies, 2(2), pp. 42-50. Recuperado de https://www.researchgate.net/publication/287984420_Prensa_nativa_digital_y_redes_ sociales_cibermedios_en_Facebook_y_Twitter

Martos, A. (2010). Redes Sociales. Madrid, España: Anaya Multimedia.

Mayoral, J. y Edo, C. (2014) Tipología de vídeos en el periodismo digital español: análisis cualitativo de cinco cibermedios. Doxa Comunicación, 19, pp. 31-56. doi: https://doi. org/10.31921/doxacom.n19a2

McHaney, R. (2012). The New Digital Shoreline: How 2.0 and Milennials Are Revolutionizing Higher Education. Sterling, Estados Unidos: Stylus Publishing.

Mcnamara, J. (2013). Public communication practices in the Web 2.0-3.0 mediascape: The case of PRevolution. Prism, 7(3), pp. 1-13. Recuperado de https://www.prismjournal.org/ uploads/1/2/5/6/125661607/v7-no3-a3.pdf

Monasterio, A. (2017). Ética algorítmica: Implicaciones éticas de una sociedad cada vez más gobernada por algoritmos. Dilemata, 24, 185-217. Recuperado de https://www.dilemata. net/revista/index.php/dilemata/article/view/412000107

Newman, N. (2009. The rise of social media and its impact on mainstream journalism (Reuters Institute for the Study of Journalism: Working Papers). Oxford, Reino Unido: Reuters Institute for the Study of Journalism.

Noguera, J.M. (2010). Redes sociales como paradigma periodístico. Medios españoles en Facebook. Revista Latina de Comunicación Social, 65, pp. 176-186. doi: https://doi.org/10.4185/ RLCS-65-2010-891-176-186

O'Reilly, T. (2005). What is Web 2.0. Design Patterns and Business Models for the Next Generation of Software. Oreilly.net. Recuperado de https://www.oreilly.com/pub/a/web2/archive/ what-is-web-20.html

Parra, D. (2017). La apuesta de los nativos digitales españoles por los géneros periodísticos de análisis: los casos de Ctxt y Bez Diario. Congreso Universitario Internacional sobre la Comunicación en la Profesión y en la Universidad de Hoy. Madrid (25-26 octubre). 
Parra, D. y Martínez Arias, S. (2018) Tecnologías de la Gestión Periodística de la Información Digital. Conceptos básicos. Madrid, España: Creative Commons.

Peña, S., Lazkano, I. y García González, D. (2016). La transición digital de los diarios europeos: nuevos productos y nuevas audiencias. Comunicar, 46(24), pp. 27-36. doi: http://dx.doi. org/10.3916/C46-2016-03

Peñafiel, C. (2016). Reinvención del periodismo en el ecosistema digital y narrativas transmedia. adComunica. Revista Científica de Estrategias, Tendencias e Innovación en Comunicación, 12, pp. 163-182. doi: http://dx.doi.org/10.6035/2174-0992.2016.12.10

Pérez Soler, S. (2018). Periodismo y redes sociales. Claves para la gestión de contenidos digitales. Barcelona, España: UOC.

Pujol, F. (2019). La prensa en Twitter: análisis de actividad e impacto en 2018. Cuadernos de Periodistas, 38, pp. 54-67. Recuperado de http://www.cuadernosdeperiodistas.com/ media/2019/10/Francesc-puyol_compressed.pdf

Rodríguez Martínez, R., Codina, L. y Pedraza, R. (2010). Cibermedios y web 2.0: modelo de análisis y resultados de aplicación. El Profesional de la Información, 19(1), pp. 35-44. doi: https://doi.org/10.3145/epi.2010.ene.05

Rojas, J.L. y Marín, C.R. (2016). Modelos de negocio para el periodismo deportivo de nicho en el contexto postindustrial. Ámbitos, 33. Recuperado de http://institucional.us.es/ambitos/?p=2484

Salaverría, R. (2010). ¿Ciberperiodismo sin periodistas? Diez ideas para la regeneración de los profesionales de los medios digitales. En F. Campos, (Coord.), El cambio mediático, pp. 236-249. Salamanca, España: Comunicación Social.

Salaverría, R. (2017) Tipología de los cibermedios periodísticos: bases teóricas para su clasificación. Revista Mediterránea de Comunicación, 8(1), pp. 19-32. doi: https://www.doi. org/10.14198/MEDCOM2017.8.1.2

Schapals, A., Maeres, P. y Hanusch, F. (2019). Working on the Margins: Comparative Perspectives on the Roles and Motivations of Peripherical Actors in Journalism. Media and Communication, 7(4), pp. 19-30. doi: https://doi.org/10.17645/mac.v7i4.2374

Steiner, C. (2012). Automate This: How Algorithms Came to Rule The World. Nueva York, Estados Unidos: Portfolio / Penguin.

Tapscott, D. (1995). The Digital Economy. Promise and Peril in The Age of Networked Intelligence. Nueva York, Estados Unidos: McGraw-Hill.

Thorsen, E. (2017). Cryptic Journalism. News reporting of encryption. Digital Journalism, 5(3), pp. 299-317. doi: https://doi.org/10.1080/21670811.2016.1243452

Thurman, N. (2018). Social Media, Surveillance, and News Work. Digital Journalism, 6(1), pp. 76-97. doi: https://doi.org/10.1080/21670811.2017.1345318

Toffler, A. (1980). Powershift Knowledge, Wealth and Violence at the Edge of the 21st Century. Nueva York, Estados Unidos: Bantam.

Ufarte, M.J. y Murcia, F.J. (2018). El fact checking: en busca de un nuevo modelo de negocio sostenible para el periodismo. Estudio de caso de Miniver. Miguel Hernández Communication Journal, 9(2), pp. 511-534. doi: http://dx.doi.org/10.21134/mhcj.v0i9.267

Vara, A. (2016) El Confidencial: innovar en contenido como clave del éxito. En C. Sádaba (Coord.), Innovación y desarrollo de los cibermedios en España, pp. 166-177. Pamplona, España: EUNSA.

Vázquez Herrero, J. (2018). Los laboratorios periodísticos: estudio de caso de El Confidencial. VI Congreso Internacional de la AEIC, Comunicación y Conocimiento. Salamanca (26-29 junio), pp. 2109-2122. Recuperado de https://www.researchgate.net/publication/328215478_Los_ laboratorios_periodisticos_estudio_de_caso_de_El_Confidencial

Wihbey, J., Joseph, K. y Lazer, D. (2019). The social silos of journalism? Twitter, news media and partisan segregation. New Media E Society, 21(4), pp. 815-835. doi: https://doi. org/10.1177/1461444818807133 